\title{
The angiogenesis regulator vasohibin-1 inhibits ovarian cancer growth and peritoneal dissemination and prolongs host survival
}

\author{
YOSHIFUMI TAKAHASHI $^{1}$, YASUSHI SAGA ${ }^{1}$, TAKAHIRO KOYANAGI ${ }^{1}$, YUJI TAKEI $^{1}$, \\ SIZUO MACHIDA $^{1}$, AKIYO TANEICHI ${ }^{1}$, HIROAKI MIZUKAMI ${ }^{2}$, YASUFUMI SATO ${ }^{3}$, \\ SHIGEKI MATSUBARA ${ }^{1}$ and HIROYUKI FUJIWARA ${ }^{1}$ \\ ${ }^{1}$ Department of Obstetrics and Gynecology, and ${ }^{2}$ Division of Genetic Therapeutics, Center for Molecular Medicine, \\ School of Medicine, Jichi Medical University, Tochigi; ${ }^{3}$ Department of Vascular Biology, \\ Institute of Development, Aging, and Cancer, Tohoku University, Sendai, Japan
}

Received July 29, 2015; Accepted September 18, 2015

DOI: $10.3892 /$ ijo.2015.3193

\begin{abstract}
Vasohibin-1 (VASH1) is expressed in vascular endothelial cells stimulated by several angiogenic growth factors and displays autocrine activity to regulate angiogenesis via a negative feedback mechanism. In this study, we investigated the effect of VASH1 on ovarian cancer progression using VASH1-expressing ovarian cancer cells in vitro and in vivo. The growth ability of ovarian cancer cells engineered to express the VASH1 gene remained unchanged in vitro. However, we showed that VASH1 secretion by tumor cells inhibited the growth of human umbilical vein endothelial cells. Further, animal experiments showed that VASH1 expression inhibited tumor angiogenesis and growth. In a murine model of peritoneal dissemination of ovarian cancer cells, VASH1 inhibited peritoneal dissemination and ascites, resulting in significantly prolonged survival in mice. This indicates that VASH1 exerts an antitumor effect on ovarian cancer by inhibiting angiogenesis in the tumor environment. These findings suggest that a novel therapy based on VASH1 could be a useful therapeutic strategy for ovarian cancer.
\end{abstract}

\section{Introduction}

Ovarian cancer is the second most common malignant tumor in gynecology and is the fifth leading cause of cancer-related mortality in women (1). Since early ovarian cancer is often asymptomatic, more than half of patients are initially diagnosed at advanced disease stages with peritoneal dissemination and ascites (2). The standard therapy for advanced ovarian cancer is a combination of cytoreductive surgery and platinum/taxane-

Correspondence to: Dr Yasushi Saga, Department of Obstetrics and Gynecology, School of Medicine, Jichi Medical University, 3311-1 Yakushiji, Shimotsuke, Tochigi 329-0498, Japan

E-mail: saga@jichi.ac.jp

Key words: angiogenesis, ovarian cancer, peritoneal dissemination, SKOV-3, vasohibin-1 based chemotherapy. Ovarian cancer is relatively sensitive to chemotherapy and many patients achieve remission with this combinatorial therapy $(3,4)$. However, the benefit is temporary, and more than half of these patients develop recurrence and die of the disease. Thus, there are limitations to current treatment options, necessitating the development of novel treatment strategies.

Angiogenesis is a phenomenon in which new blood vessels develop from the existing vasculature. Normal angiogenesis is a vital reaction observed only in strictly controlled situations, such as organogenesis during fetal development, the endometrial proliferative stage, and the wound healing process. However, pathological angiogenesis is closely associated with the progression of proliferative diseases (such as diabetic retinopathy and rheumatoid arthritis) and solid tumors. In 1971, Folkman proposed the theory that the growth of tumor cells depends on angiogenesis and that inhibition of angiogenesis is a useful antitumor strategy (5). Subsequently, many studies have been published describing the regulatory mechanisms of tumor angiogenesis and related processes.

Angiogenesis is regulated by a balance between angiogenic growth factors, such as VEGF (6) and FGF (7), and angiogenesis inhibitors, such as angiostatin (8), endostatin (9), pigment epithelium-derived factor (10), $16 \mathrm{kDa}$ prolactin (11), and thrombospondin-1 (12). In 2004, the novel angiogenesis inhibitor vasohibin-1 (VASH1) produced by human umbilical vein endothelial cells (HUVEC) stimulated by VEGF and FGF was identified (13). VASH1 is expressed in VEGF-stimulated vascular endothelial cells and acts on vascular endothelial cells themselves via a negative feedback mechanism that regulates angiogenesis $(13,14)$. Mouse corneal assays have also confirmed that VASH1 has inhibitory effects on pathological angiogenesis caused by several angiogenic factors, including VEGF, FGF, and PDGF $(13,15)$, suggesting that the targets of the anti-angiogenic action are wide-ranging. VASH1 lacks a classical secretory signal and its secretion mechanism was previously unknown. However, it was recently discovered that VASH1 binds small vasohibin-binding protein (SVBP), thereby enabling its secretion (16). VASH1 has been associated with diseases involving abnormal angiogenesis, including malignant tumors (17-21), atherosclerosis (22), diabetic retinopathy 
(23), diabetic nephropathy $(24,25)$, and age-related macular degeneration $(26,27)$. It has also been shown that VASH1 inhibits lymphangiogenesis and lymph node metastasis as well as angiogenesis (15). VASH1 could be effective for the treatment of various diseases, including malignant tumors. Animal experiments using murine lung cancer cell lines have demonstrated the anti-angiogenic and antitumor activity of VASH1 (13). In this study, we investigated the previously unreported antitumor effect of VASH1 on ovarian cancer in an experimental animal model of ovarian cancer.

\section{Materials and methods}

Cell lines and culture. The human ovarian cancer cell line SKOV-3 (28) was purchased from the American Type Culture Collection (Manassas, VA, USA). The SKOV-3 cell line, which has previously been reported to produce VEGF $(29,30)$, was cultured in Dulbecco's modified Eagle's medium/F12 (DMEM/F12) medium (Life Technologies, Carlsbad, CA, USA) containing 10\% fetal calf serum (SigmaAldrich, St. Louis, MO, USA) and $1 \%$ penicillin/streptomycin (Life Technologies). HUVECs were obtained from Kurabo Industries, Ltd. (Osaka, Japan) and cultured in a 10-cm, type 1 collagen-coated culture dish (Asahi Glass Co., Ltd., Tokyo, Japan) containing EBM-2 medium (Lonza, Walkersville, MD, USA) supplemented with EGM-2-MV-SingleQuots (Lonza), VEGF, FGF, insulin-like growth factor-1, epidermal growth factor, and $2 \%$ fetal calf serum. All cells were cultured at $37^{\circ} \mathrm{C}$ in a $5 \%$ carbon dioxide atmosphere.

Establishment of a VASH1-expressing cell line. Human VASH1 cDNA (NCBI database; Gene ID 22846) was inserted into the cloning site (SmaI, XbaI) of pCMV-IRES-bsr (31) to generate a VASH1 expression vector. The VASH1 expression vector or the control plasmid vector expressing luciferase [pCMV-LUC-IRES-bsr (31)] was transfected into SKOV-3 cells using Lipofectamine LTX Plus reagent (Invitrogen, Carlsbad, CA, USA) according to the manufacturer's instructions. Subsequently, the transfected cells were selectively cultured in medium containing blasticidin $\mathrm{S} \mathrm{HCl}$ (Funakoshi Co., Ltd., Tokyo, Japan) and blasticidin-resistant cells were obtained. These cells were cloned by limiting dilution and VASH1-expressing and control clones were established.

Quantitative reverse transcriptase-polymerase chain reaction (RT-PCR). Extraction of mRNA from cultured cells was performed using an RNeasy Mini kit (Qiagen, Valencia, CA, USA) according to the manufacturer's instructions. The mRNA was subjected to quantitative RT-PCR using a One Step SYBR PrimeScript PLUS RT-PCR kit (Perfect RealTime, Takara, Otsu, Japan) and Thermal Cycler Dice Real Time System II (Takara) according to the manufacturer's protocol. The amount of each mRNA was expressed as a ratio of the glyceraldehyde 3-phosphate dehydrogenase (GAPDH)corrected fluorescent signal intensity. The primer sequences were as follows: GAPDH forward, ACCACAGTCCATGCC ATCAC; reverse, GGGCCTCTTTGGTCATTTCC; VASH1 forward, AGATCCCCATACCGAGTGTG; reverse, GGGCCT CTTTGGTCATTTCC.
In vitro cell growth. A total of $1 \times 10^{3}$ cancer cells were seeded in each well of a 96-well plate and an XTT assay kit (Roche Diagnostics, Mannheim, Germany) was used every $24 \mathrm{~h}$. The absorbance was measured $24 \mathrm{~h}$ later at $490 \mathrm{~nm}$, and cell growth curves were generated.

Endothelial cell growth in vitro. A total of $1 \times 10^{6}$ cancer cells were seeded in each well of a 6-well plate and cultured in EBM-2 medium containing no growth factors. The culture supernatant was collected $24 \mathrm{~h}$ later. A total of $2 \times 10^{3}$ HUVEC were seeded in each well of a 96-well plate and cultured using the culture supernatant. An XTT assay kit was used after $48 \mathrm{~h}$ and the absorbance was measured $24 \mathrm{~h}$ later at $490 \mathrm{~nm}$.

Western blotting. Cells were lysed using a lysis buffer (1\% NP-40, $150 \mathrm{mM} \mathrm{NaCl}$, and $50 \mathrm{mM}$ Tris-HCl, $\mathrm{pH} 8.0$ ), and proteins were extracted. These samples were mixed with an SDS sample buffer [10 mM Tris- $\mathrm{HCl}$ (pH 7.5), $150 \mathrm{mM}$ $\mathrm{NaCl}, 1 \% \mathrm{SDS}$, and EDTA-free proteinase inhibitor cocktail (Roche)]; the mixtures were electrophoresed on $10 \%$ polyacrylamide gels, followed by transfer to polyvinylidene fluoride membranes (Merck Millipore, Ltd., Billerica, MA, USA). The membranes were incubated at room temperature for $1 \mathrm{~h}$ in Tris buffer ( $\mathrm{pH}$ 7.6) containing 5\% skimmed milk (Wako Pure Chemical Industries, Ltd., Tokyo, Japan), and were incubated at $4^{\circ} \mathrm{C}$ overnight with $1 \mu \mathrm{g} / \mathrm{ml}$ of mouse monoclonal anti-VASH1 (13) or rabbit anti-actin antibody (Sigma-Aldrich). After washing three times with phosphate-buffered salineTween-20 (PBS-T), the membranes were incubated with peroxidase-conjugated anti-mouse or anti-rabbit IgG antibody (GE Healthcare, Buckinghamshire, UK) at room temperature for $1 \mathrm{~h}$. After washing with PBS-T three times, the membranes were subjected to chemiluminescence using an ECL kit (American Biosciences, Piscataway, NJ, USA) and chemiluminescence was detected using a cooled CCD camera system (LAS-4000 mini, GE Healthcare).

Animal experiments. Four- to six-week-old BALB/c nude mice (Clea Japan, Tokyo, Japan) were kept in a specific pathogenfree environment. All animal experiments followed the Guidelines for Animal Experiments of Tohoku University and Jichi Medical University.

Subcutaneous tumor transplant model. Nude mice were subcutaneously transplanted with $5 \times 10^{6}$ tumor cells. The tumors formed were measured with a caliper and tumor volumes [long diameter x (short diameter) ${ }^{2}$ x 1/2] were calculated.

Peritoneal dissemination model and survival time. A total of $5 \times 10^{6}$ cancer cells were used to inoculate the peritoneal cavity of nude mice, and the amount of ascitic fluid and number of tumor nodules on the surface of the intestine and mesentery were measured. Mouse survival was determined twice per day and survival curves were constructed using the Kaplan-Meier method.

Immunohistochemical staining. Subcutaneous tumor transplant model mice were sacrificed using isoflurane inhalation. The tumor was removed, embedded in OCT compound (Sakura Finetek Japan Co., Ltd., Tokyo, Japan) and frozen. 
A

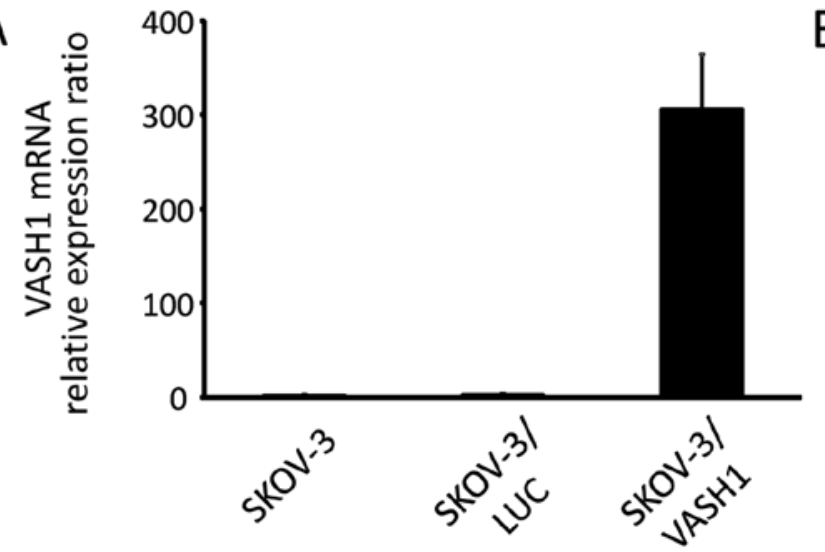

B
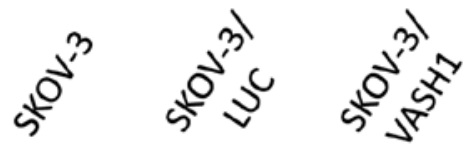

VASH1

Actin

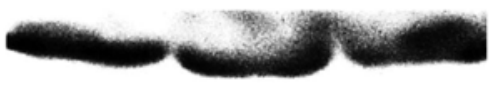

Figure 1. Establishment of a VASH1-expressing cell line. (A) Quantitative RT-PCR of VASH1. (B) Western blotting results. Expression of VASH1 mRNA and protein was only observed in SKOV-3/VASH1.

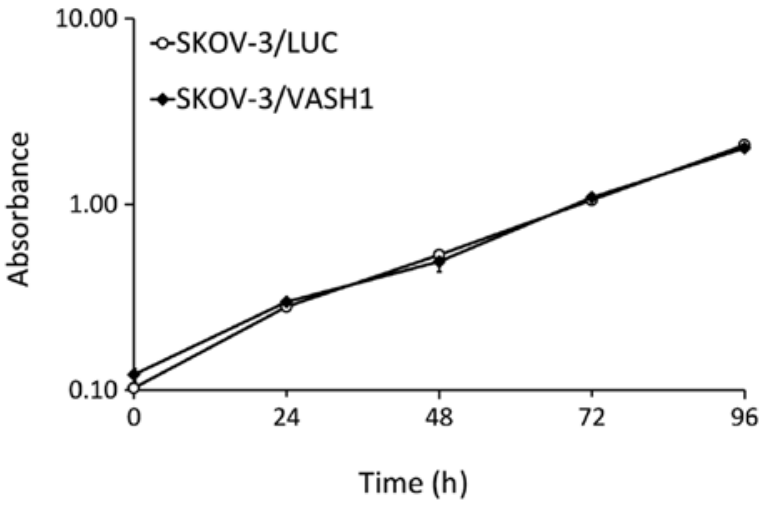

Figure 2. Cell growth curves of SKOV-3/LUC and SKOV-3/VASH1 in vitro. No significant difference was observed between groups.

Sections $7-\mathrm{mm}$ thick were cut, fixed in methanol at $-20^{\circ} \mathrm{C}$ for $20 \mathrm{~min}$, blocked with $1 \%$ BSA at room temperature for $30 \mathrm{~min}$, and incubated with a 1:500 dilution of anti-mouse CD31 antibody (Research Diagnosis, Flanders, NJ, USA) overnight at $4^{\circ} \mathrm{C}$. After washing three times with PBS, sections were incubated with a 1:500 dilution of Alexa 488-conjugated anti-rat IgG antibody (Molecular Probes, Eugene, OR, USA) at room temperature for $1 \mathrm{~h}$, washed three times with PBS and embedded in fluorescent mounting medium. Specimens were analyzed using a BZ-9000 fluorescence microscope (Keyence, Osaka, Japan). The vascular area was calculated as the average blood vessel area in five microscopic fields. Quantitative analysis was performed using BZ-HIC software (Keyence).

Statistics. Student's t-test was used for comparison between two groups. The log-rank test was used to compare Kaplan-Meier survival curves. p-values $<0.05$ were considered significant.

\section{Results}

Establishment of a VASH1-expressing cell line. Fig. 1 shows the results for VASH1 expression analyzed using quantitative RT-PCR and western blotting, respectively, for SKOV-3, SKOV-3/LUC, and SKOV-3/VASH1. Expression of VASH1 mRNA and protein was only observed in SKOV-3/VASH1,

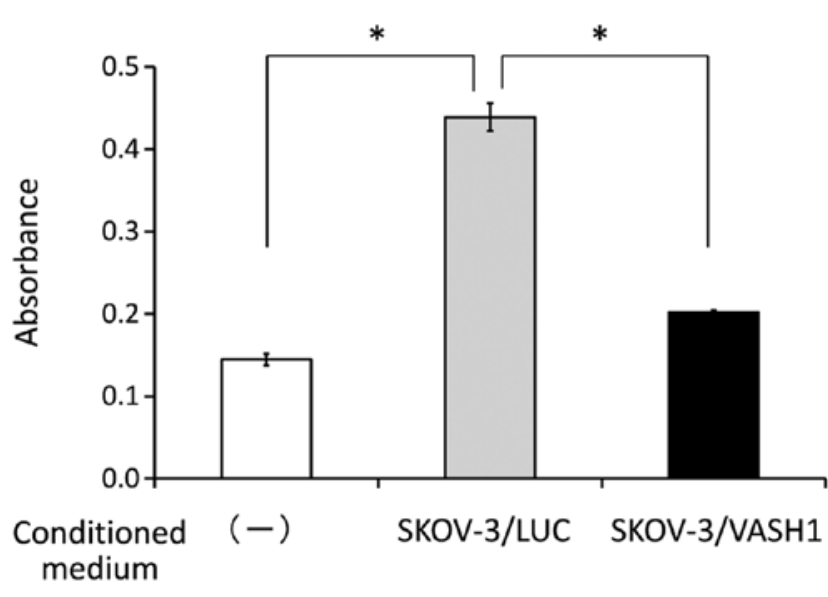

Figure 3. HUVEC number after supplementation with culture supernatant. The absorbance was significantly higher in the group supplemented with the SKOV-3/LUC culture supernatant than in the non-supplemented group but significantly lower in the group supplemented with the SKOV-3/ VASH1 culture supernatant compared with the group supplemented with the SKOV-3/LUC supernatant. " $\mathrm{p}<0.01$.

indicating the establishment of the VASH1-expressing ovarian cancer cell line SKOV-3.

Effect of VASHI on cell growth in vitro. The effects of SKOV-3/LUC and SKOV-3/VASH1 on cell growth in vitro were compared. As shown in Fig. 2, there was no significant difference in the cell growth curve, indicating that VASH1 expression did not influence the growth of SKOV-3 cells in vitro.

The effect of VASHI secretion by SKOV-3/VASHI on HUVEC. The effect of VASH1 secretion by SKOV-3/VASH1 on HUVEC was examined. As shown in Fig. 3, the absorbance indicating the number of HUVEC $48 \mathrm{~h}$ later was significantly higher in the group supplemented with the culture supernatant of SKOV-3/LUC $(n=3)$ than in the non-supplemented group $(n=3)(0.44 \pm 0.02$ vs. $0.15 \pm 0.01$, respectively; $p<0.01)$, but was significantly lower in the group supplemented with the culture supernatant of SKOV-3/VASH1 $(n=3)$ than in the group supplemented with the supernatant of SKOV-3/LUC 
A

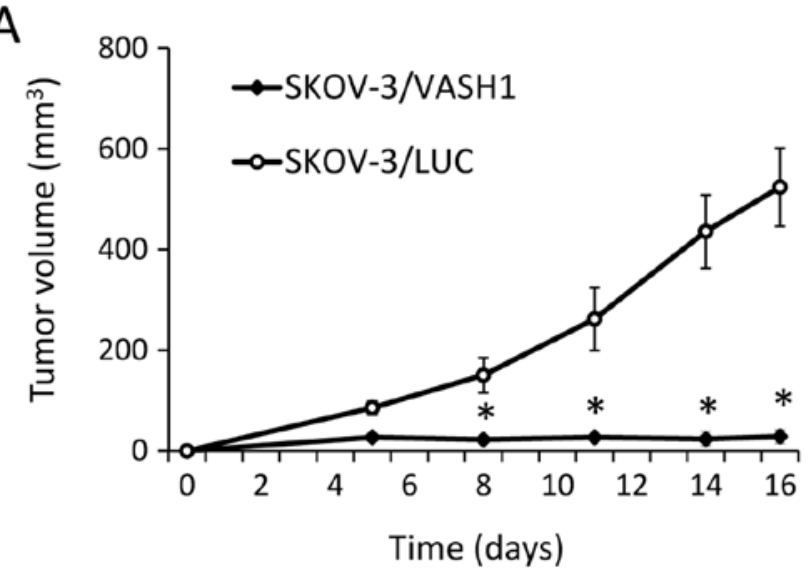

c

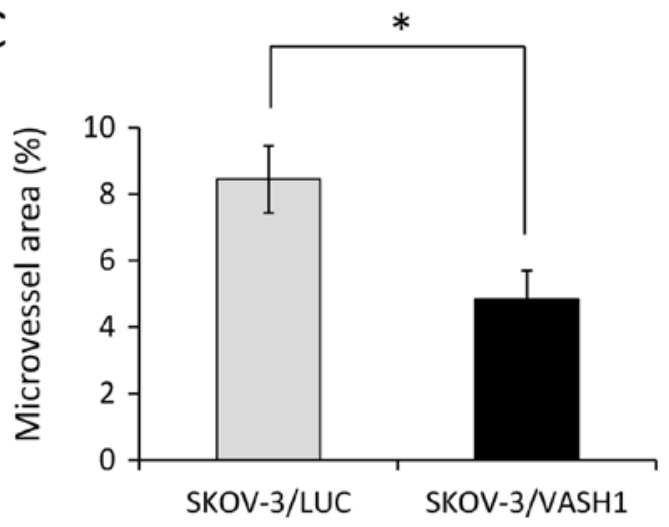

B

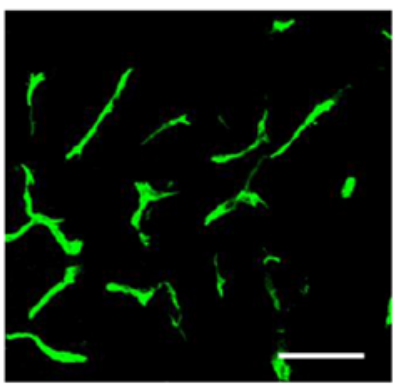

SKOV-3/LUC

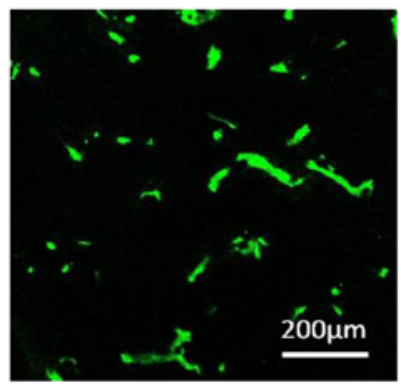

SKOV-3/VASH1

Figure 4. Anti-angiogenic and antitumor activity of VASH1. (A) Growth curves of SKOV-3/LUC and SKOV-3/VASH1 subcutaneous tumors. (B) Immunohistochemical staining of new blood vessels in tumor tissue. (C) The microvessel area in SKOV-3/VASH1 tumors per high-power field was significantly lower than that in SKOV-3/LUC tumors. "p $<0.01$.

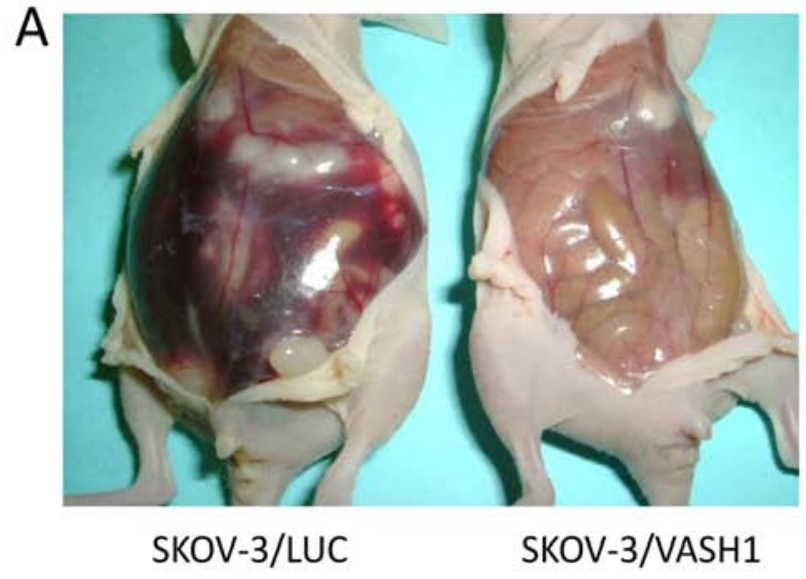

B

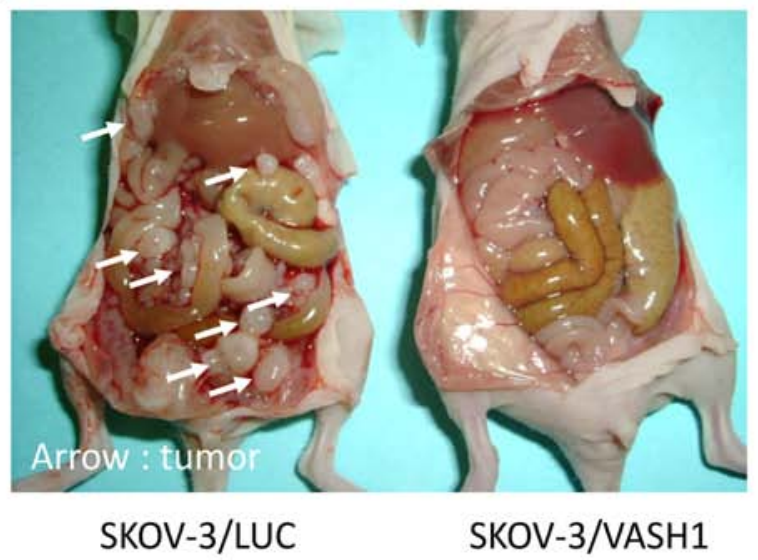

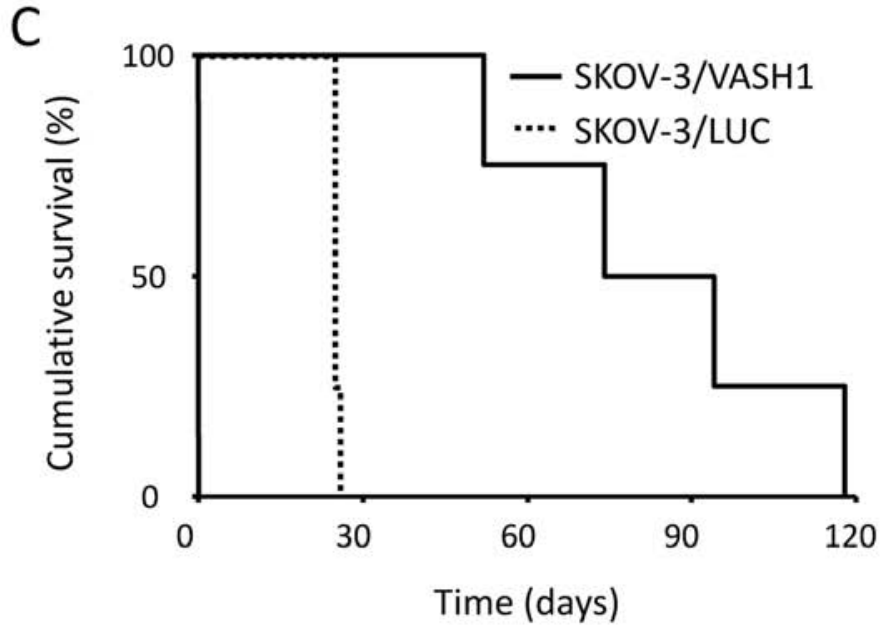

Figure 5. Peritoneal dissemination-inhibitory effect of VASH1. (A and B) Findings at laparotomy 21 days post-intraperitoneal inoculation. (C) Survival curves of mice with intraperitoneal transplants. 
$(n=3)(0.20 \pm 0.002$ vs. $0.44 \pm 0.02$, respectively; $p<0.01)$. This indicates that VASH1 secreted by SKOV-3/VASH1 inhibited the growth of HUVEC in vitro.

Antitumor and anti-angiogenic effects of VASH1. Fig. 4A shows the growth curves of SKOV-3/LUC and SKOV-3/VASH1 subcutaneous tumors. Both SKOV-3/LUC and SKOV-3/ VASH1 groups formed tumor nodules 1 week after inoculation. Subsequently, tumor enlargement was observed in the SKOV-3/LUC group but not in the SKOV/VASH1 group. Thus, VASH1 expression inhibited the subcutaneous growth of SKOV-3 tumors in vivo. Next, new blood vessel formation in resected SKOV-3/LUC and SKOV-3/VASH1 tumors was evaluated using immunohistochemical staining (Fig. 4B). The microvessel density in SKOV-3/VASH1 tumors $(n=3)$ per high-power field $(4.9 \pm 0.9 \%)$ was significantly lower than that in SKOV-3/LUC tumors $(\mathrm{n}=3)(8.5 \pm 1.0 \%, \mathrm{p}<0.01)$ (Fig. 4 C). Thus, VASH1 expression inhibited angiogenesis and growth in SKOV-3 tumors.

Peritoneal dissemination-inhibiting effect of VASH1. The effect of VASH1 expression on ascites and peritoneal dissemination was studied in murine models of peritoneally disseminated ovarian cancer. Fig. 5A and B show findings at laparotomy 21 days after the inoculation of the peritoneal cavity of nude mice with SKOV-3/LUC or SKOV-3/VASH1 cells. Marked ascites (Fig. 5A, left) and many tumor nodules disseminated in the peritoneum (Fig. 5B, left) were observed in SKOV-3/LUC-inoculated mice but little or no ascites or peritoneal dissemination was observed in SKOV-3/VASH1inoculated mice (Fig. 5A, right and B, right). The number of disseminated tumor nodules on the surface of the intestine and mesentery was significantly lower in SKOV-3/VASH1inoculated $(n=4)$ than in SKOV-3/LUC-inoculated $(n=4)$ mice ( $3 \pm 4$ vs. $87 \pm 48$ nodules, respectively; $p<0.01$ ). Thus, VASH1 expression inhibited peritoneal dissemination of SKOV-3 cells.

Next, the survival of SKOV-3/LUC- and SKOV-3/VASH1inoculated mice was examined. Survival curves are shown in Fig. 5C. All mice in the SKOV-3/LUC group $(n=4)$ died by day 28 post-inoculation, whereas survival of SKOV-3/VASH1 mice $(n=4)$ was significantly increased $(\mathrm{p}<0.01)$. Thus, VASH1 expression inhibited the peritoneal dissemination of SKOV-3 cells, significantly prolonging mouse survival.

\section{Discussion}

Animal studies using lung or breast cancer cell lines have reported the anti-angiogenic and antitumor activity of VASH1 $(13,15,17)$. However, no studies have reported its effects on ovarian cancer, which spreads by peritoneal dissemination, a unique pattern of tumor progression. Therefore, we investigated the antitumor effect of VASH1 on ovarian cancer in murine models of peritoneally disseminated ovarian cancer.

In this study, the growth ability of ovarian cancer cells engineered to express the VASH1 gene remained unchanged. An experiment using HUVECs showed that VASH1 secreted by tumor cells inhibited the growth of vascular endothelial cells. Next, animal experiments showed that VASH1 expression inhibited angiogenesis in tumors. In addition, in a murine model of peritoneal dissemination of cancer cells, VASH1 inhibited peritoneal dissemination and ascites, thus significantly prolonging survival. This indicates that VASH1 exerts an antitumor effect on ovarian cancer by inhibiting angiogenesis in the tumor environment.

In recent years, molecular-targeted therapy against VEGF has been clinically applied and has demonstrated some effectiveness. Bevacizumab, an anti-VEGF monoclonal antibody, has been widely used in the clinical setting in the treatment of colorectal cancer, non-small cell lung cancer, and breast cancer. In addition, a phase III clinical trial of bevacizumab for ovarian cancer showed that the progression-free survival time was significantly higher in a group of patients treated with bevacizumab and chemotherapy followed by continued bevacizumab monotherapy than in a group of patients treated with chemotherapy alone (32). Furthermore, large-scale clinical studies reported similar midterm results $(33,34)$, supporting the efficacy of bevacizumab therapy for ovarian cancer. However, a phenomenon called 'evasive resistance', which is observed in tumors after anti-VEGF therapy, has attracted attention (35). Proposed mechanisms for the acquisition of this resistance include the induction of angiogenic factors other than VEGF, mobilization of bone marrow-derived endothelial progenitor cells, covering of tumor vascular endothelial cells by pericytes, and an increase in the invasive capacity of tumor cells. An animal experiment using an antibody targeting the VEGF receptor (VEGFR) 2 showed that vascular regression and tumor reduction occurred first, followed by the induction of angiogenesis, leading to tumor regrowth (36). Analysis of the mRNA of regrown tumors revealed increased expression of angiogenic factors other than VEGF, such as FGF, ephrin, and angiopoietin (36). In addition, a clinical study reported that the treatment of glioblastoma patients with VEGFR inhibitors resulted in increased serum FGF levels (37). Since various angiogenic factors are involved in tumor angiogenesis, there are limitations to treatment targeting a single angiogenic factor. Therefore, to achieve sufficient therapeutic benefit, it may be necessary to simultaneously target multiple angiogenic factors. In the above-cited study, the combined use of anti-VEGFR2 antibodies and soluble FGF receptor (an FGF antagonist) successfully delayed tumor regrowth (36). In our study, VASH1 inhibited angiogenesis mediated by various angiogenic factors other than VEGF $(13,15)$. These observations suggest that VASH1, which acts alone to inhibit multiple angiogenic factors, is a more effective therapeutic agent compared with VEGF inhibitors in terms of overcoming evasive resistance.

Physiologically, VASH1 is induced in vascular endothelial cells to prevent excessive angiogenesis associated with VEGF stimulation. The application of VASH1 to the treatment of diseases caused by abnormal angiogenesis requires doses that far exceed the physiological VASH1 expression (38). To date, the local administration of purified VASH1 protein preparations $(27,38)$ and local $(38,39)$ or systemic $(15,17,22,24,25)$ administration of an adenovirus vector encoding VASH1 have been attempted. We previously reported the efficacy of therapeutic strategies using adeno-associated virus (AAV) vectors carrying various therapeutic factors $(40,41)$. AAV vectors have advantages, in that they are derived from non-pathogenic viruses with a strong safety profile; also, non-dividing cells, such as neurons and muscle cells, can also be efficiently 
transfected, with transfected genes expressed over a prolonged period of time (42). If these advantages were exploited, it would be possible to transfect skeletal muscle cells with therapeutic genes using AAV vectors carrying therapeutic factors to induce muscle cells expression and secretion of these factors into the blood and to deliver them to target lesions. Using an animal model, we demonstrated the feasibility of inhibiting ovarian cancer progression by constructing an AAV vector containing soluble VEGFR-1 (sFlt-1), a VEGF antagonist, and injecting this vector into skeletal muscle (40). Using a similar strategy, we showed that an AAV vector carrying soluble VEGFR-3 (sFlt-4), an antagonist of VEGF-C that promotes tumor lymphangiogenesis and lymphatic metastasis, successfully inhibited lymph node metastasis of endometrial carcinoma (41). A gene therapy strategy using vectors such as AAV is a promising application of VASH1 therapy. In this case, since SVBP is required for the secretion of VASH1, co-transfection of VASH1 and SVBP expression vectors could be effective in increasing VASH1 secretion into the blood.

Unlike sFlt-1 and sFlt-4, the underlying inhibitory mechanism of VASH1 is still unknown. We are currently trying to elucidate this mechanism. If this mechanism is understood in future, it might be possible to identify patients who will benefit from VASH1.

Anti-angiogenic therapy is now approved for several types of cancers and drugs targeting the VEGF signals are in clinical use. However, such drugs can have side effects that include hypertension and proteinuria due to the impairment of normal quiescent vessels. It was recently reported that VASH1 protects endothelial cells from premature senescence and stress-induced cell death via the induction of superoxide dismutase 2 and sirtuin 1 (43). Animal experiments demonstrated that VASH1 did not increase mean blood pressure and urinary albumin excretion (44). It has also been reported that VASH1 does not affect any morphological changes in normal blood vessels (15), wound healing, body weight, and peripheral blood flow (45) in adenoviral VASH1 gene-treated mice. These findings suggest that VASH1 can be a potential candidate for anti-angiogenic treatment.

In conclusion, this study showed that VASH1, a negative feedback regulator of angiogenesis, inhibited tumor angiogenesis and peritoneal dissemination in ovarian tumors, thereby prolonging survival in mouse models of ovarian cancer. These results suggest that a novel therapy based on VASH1 could be a very useful therapeutic strategy for ovarian cancer.

\section{Acknowledgements}

This study was supported by The Reserch Award to Jichi Medical University Graduate Student (Y.T.).

\section{References}

1. Siegel R, Naishadham D and Jemal A: Cancer statistics, 2012. CA Cancer J Clin 62: 10-29, 2012.

2. Heintz AP: Surgery in advanced ovarian carcinoma: Is there proof to show the benefit? Eur J Surg Oncol 14: 91-99, 1988.

3. McGuire WP, Hoskins WJ, Brady MF, Kucera PR, Partridge EE, Look KY, Clarke-Pearson DL and Davidson M: Cyclophosphamide and cisplatin compared with paclitaxel and cisplatin in patients with stage III and stage IV ovarian cancer. $\mathrm{N}$ Engl J Med 334: 1-6, 1996.
4. Takei Y, Suzuki M, Ohwada M, Saga Y, Kohno T, Machida S and Sato I: A feasibility study of paclitaxel and carboplatin therapy in Japanese patients with epithelial ovarian cancer. Oncol Rep 10: 951-955, 2003.

5. Folkman J: Tumor angiogenesis: Therapeutic implications. N Engl J Med 285: 1182-1186, 1971.

6. Leung DW, Cachianes G, Kuang WJ, Goeddel DV and Ferrara N: Vascular endothelial growth factor is a secreted angiogenic mitogen. Science 246: 1306-1309, 1989.

7. FahmyRG,Dass CR,SunLQ, ChestermanCN and KhachigianLM: Transcription factor Egr-1 supports FGF-dependent angiogenesis during neovascularization and tumor growth. Nat Med 9: 1026-1032, 2003.

8. O'Reilly MS, Holmgren L, Shing Y, Chen C, Rosenthal RA, Moses M, Lane WS, Cao Y, Sage EH and Folkman J: Angiostatin: A novel angiogenesis inhibitor that mediates the suppression of metastases by a Lewis lung carcinoma. Cell 79: 315-328, 1994.

9. O'Reilly MS, Boehm T, Shing Y, Fukai N, Vasios G, Lane WS, Flynn E, Birkhead JR, Olsen BR and Folkman J: Endostatin: An endogenous inhibitor of angiogenesis and tumor growth. Cell 88: 277-285, 1997.

10. Dawson DW, Volpert OV, Gillis P, Crawford SE, Xu H, Benedict W and Bouck NP: Pigment epithelium-derived factor: A potent inhibitor of angiogenesis. Science 285: 245-248, 1999.

11. Clapp C, Martial JA, Guzman RC, Rentier-Delure F and Weiner RI: The 16-kilodalton N-terminal fragment of human prolactin is a potent inhibitor of angiogenesis. Endocrinology 133: 1292-1299, 1993.

12. Tolsma SS, Volpert OV, Good DJ, Frazier WA, Polverini PJ and Bouck N: Peptides derived from two separate domains of the matrix protein thrombospondin-1 have anti-angiogenic activity. J Cell Biol 122: 497-511, 1993.

13. Watanabe K, Hasegawa Y, Yamashita H, Shimizu K, Ding Y, Abe M, Ohta H, Imagawa K, Hojo K, Maki H, et al: Vasohibin as an endothelium-derived negative feedback regulator of angiogenesis. J Clin Invest 114: 898-907, 2004.

14. Shimizu K, Watanabe K, Yamashita H, Abe M, Yoshimatsu H, Ohta $\mathrm{H}$, Sonoda $\mathrm{H}$ and Sato Y: Gene regulation of a novel angiogenesis inhibitor, vasohibin, in endothelial cells. Biochem Biophys Res Commun 327: 700-706, 2005.

15. Heishi T, Hosaka T, Suzuki Y, Miyashita H, Oike Y, Takahashi T, Nakamura T, Arioka S, Mitsuda Y, Takakura T, et al: Endogenous angiogenesis inhibitor vasohibin1 exhibits broad-spectrum antilymphangiogenic activity and suppresses lymph node metastasis. Am J Pathol 176: 1950-1958, 2010.

16. Suzuki Y, Kobayashi M, Miyashita H, Ohta H, Sonoda H and Sato Y: Isolation of a small vasohibin-binding protein (SVBP) and its role in vasohibin secretion. J Cell Sci 123: 3094-3101, 2010.

17. Hosaka T, Kimura H, Heishi T, Suzuki Y, Miyashita H, Ohta H, Sonoda H, Moriya T, Suzuki S, Kondo T, et al: Vasohibin-1 expression in endothelium of tumor blood vessels regulates angiogenesis. Am J Pathol 175: 430-439, 2009.

18. Yoshinaga K, Ito K, Moriya T, Nagase S, Takano T, Niikura H, Yaegashi $\mathrm{N}$ and Sato Y: Expression of vasohibin as a novel endothelium-derived angiogenesis inhibitor in endometrial cancer. Cancer Sci 99: 914-919, 2008.

19. Yoshinaga K, Ito K, Moriya T, Nagase S, Takano T, Niikura H, Sasano H, Yaegashi N and Sato Y: Roles of intrinsic angiogenesis inhibitor, vasohibin, in cervical carcinomas. Cancer Sci 102: 446-451, 2011

20. Tamaki K, Moriya T, Sato Y, Ishida T, Maruo Y, Yoshinaga K, Ohuchi N and Sasano H: Vasohibin-1 in human breast carcinoma: A potential negative feedback regulator of angiogenesis. Cancer Sci 100: 88-94, 2009.

21. Tamaki K, Sasano H, Maruo Y, Takahashi Y, Miyashita M, Moriya T, Sato Y, Hirakawa H, Tamaki N, Watanabe M, et al: Vasohibin-1 as a potential predictor of aggressive behavior of ductal carcinoma in situ of the breast. Cancer Sci 101: 1051-1058, 2010.

22. Yamashita H, Abe M, Watanabe K, Shimizu K, Moriya T, Sato A, Satomi S, Ohta H, Sonoda H and Sato Y: Vasohibin prevents arterial neointimal formation through angiogenesis inhibition. Biochem Biophys Res Commun 345: 919-925, 2006.

23. Sato H, Abe T, Wakusawa R, Asai N, Kunikata H, Ohta H, Sonoda H, Sato Y and Nishida K: Vitreous levels of vasohibin-1 and vascular endothelial growth factor in patients with proliferative diabetic retinopathy. Diabetologia 52: 359-361, 2009. 
24. Nasu T, Maeshima Y, Kinomura M, Hirokoshi-Kawahara K, Tanabe K, Sugiyama H, Sonoda H, Sato Y and Makino H: Vasohibin-1, a negative feedback regulator of angiogenesis, ameliorates renal alterations in a mouse model of diabetic nephropathy. Diabetes 58: 2365-2375, 2009.

25. Saito D, Maeshima Y, Nasu T, Yamasaki H, Tanabe K, Sugiyama H, Sonoda H, Sato Y and Makino H: Amelioration of renal alterations in obese type 2 diabetic mice by vasohibin-1, a negative feedback regulator of angiogenesis. Am J Physiol Renal Physiol 300: F873-F886, 2011.

26. Wakusawa R, Abe T, Sato H, Yoshida M, Kunikata H, Sato Y and Nishida K: Expression of vasohibin, an antiangiogenic factor, in human choroidal neovascular membranes. Am J Ophthalmol 146: 235-243, 2008

27. Wakusawa R, Abe T, Sato H, Sonoda H, Sato M, Mitsuda Y, Takakura T, Fukushima T, Onami H, Nagai N, et al: Suppression of choroidal neovascularization by vasohibin-1, a vascular endothelium-derived angiogenic inhibitor. Invest Ophthalmol Vis Sci 52: 3272-3280, 2011.

28. Fogh J, Wright WC and Loveless JD: Absence of HeLa cell contamination in 169 cell lines derived from human tumors. J Natl Cancer Inst 58: 209-214, 1977.

29. Mesiano S, Ferrara N and Jaffe RB: Role of vascular endothelial growth factor in ovarian cancer: Inhibition of ascites formation by immunoneutralization. Am J Pathol 153: 1249-1256, 1998.

30. Wiener JR, Nakano K, Kruzelock RP, Bucana CD, Bast RC Jr and Gallick GE: Decreased Src tyrosine kinase activity inhibits malignant human ovarian cancer tumor growth in a nude mouse model. Clin Cancer Res 5: 2164-2170, 1999.

31. Urabe M, Hasumi Y, Ogasawara Y, Matsushita T, Kamoshita N, Nomoto A, Colosi P, Kurtzman GJ, Tobita K and Ozawa K: A novel dicistronic AAV vector using a short IRES segment derived from hepatitis C virus genome. Gene 200: 157-162, 1997.

32. Burger RA, Brady MF, Bookman MA, Fleming GF, Monk BJ, Huang H, Mannel RS, Homesley HD, Fowler J, Greer BE, et al; Gynecologic Oncology Group: Incorporation of bevacizumab in the primary treatment of ovarian cancer. N Engl J Med 365 2473-2483, 2011.

33. Perren TJ, Swart AM, Pfisterer J, Ledermann JA, PujadeLauraine E, Kristensen G, Carey MS, Beale P, Cervantes A Kurzeder C, et al; ICON7 Investigators: A phase 3 trial of bevacizumab in ovarian cancer. N Engl J Med 365: 2484-2496, 2011.

34. Aghajanian C, Blank SV, Goff BA, Judson PL, Teneriello MG, Husain A, Sovak MA, Yi J and Nycum LR: OCEANS: A randomized, double-blind, placebo-controlled phase III trial of chemotherapy with or without bevacizumab in patients with platinum-sensitive recurrent epithelial ovarian, primary peritoneal, or fallopian tube cancer. J Clin Oncol 30: 2039-2045, 2012.
35. Bergers G and Hanahan D: Modes of resistance to anti-angiogenic therapy. Nat Rev Cancer 8: 592-603, 2008.

36. Casanovas O, Hicklin DJ, Bergers G and Hanahan D: Drug resistance by evasion of antiangiogenic targeting of VEGF signaling in late-stage pancreatic islet tumors. Cancer Cell 8: 299-309, 2005.

37. Batchelor TT, Sorensen AG, di Tomaso E, Zhang WT, Duda DG, Cohen KS, Kozak KR, Cahill DP, Chen PJ, Zhu M, et al: AZD2171, a pan-VEGF receptor tyrosine kinase inhibitor, normalizes tumor vasculature and alleviates edema in glioblastoma patients. Cancer Cell 11: 83-95, 2007.

38. Shen J, Yang X, Xiao WH, Hackett SF, Sato $Y$ and Campochiaro PA: Vasohibin is up-regulated by VEGF in the retina and suppresses VEGF receptor 2 and retinal neovascularization. FASEB J 20: 723-725, 2006.

39. Zhou SY, Xie ZL, Xiao O, Yang XR, Heng BC and Sato Y: Inhibition of mouse alkali burn induced-corneal neovascularization by recombinant adenovirus encoding human vasohibin-1. Mol Vis 16: 1389-1398, 2010

40. Takei Y, Mizukami H, Saga Y, Yoshimura I, Hasumi Y, Takayama T, Kohno T, Matsushita T, Okada T, Kume A, et al: Suppression of ovarian cancer by muscle-mediated expression of soluble VEGFR-1/Flt-1 using adeno-associated virus serotype 1-derived vector. Int J Cancer 120: 278-284, 2007.

41. Takahashi K, Mizukami H, Saga Y, Takei Y, Urabe M, Kume A, Machida S, Fujiwara H, Suzuki M and Ozawa K: Suppression of lymph node and lung metastases of endometrial cancer by muscle-mediated expression of soluble vascular endothelial growth factor receptor-3. Cancer Sci 104: 1107-1111, 2013.

42. Mueller C and Flotte TR: Clinical gene therapy using recombinant adeno-associated virus vectors. Gene Ther 15: 858-863, 2008.

43. Miyashita H, Watanabe T, Hayashi H, Suzuki Y, Nakamura T, Ito S, Ono M, Hoshikawa Y, Okada Y, Kondo T, et al: Angiogenesis inhibitor vasohibin-1 enhances stress resistance of endothelial cells via induction of SOD2 and SIRT1. PLoS One 7: e46459, 2012.

44. Miyashita H, Suzuki H, Ohkuchi A and Sato Y: Mutual balance between vasohibin-1 and soluble VEGFR-1 in endothelial cells. Pharmaceuticals 4: 782-793, 2011.

45. Li D, Zhou K, Wang S, Shi Z and Yang Z: Recombinant adenovirus encoding vasohibin prevents tumor angiogenesis and inhibits tumor growth. Cancer Sci 101: 448-452, 2010. 